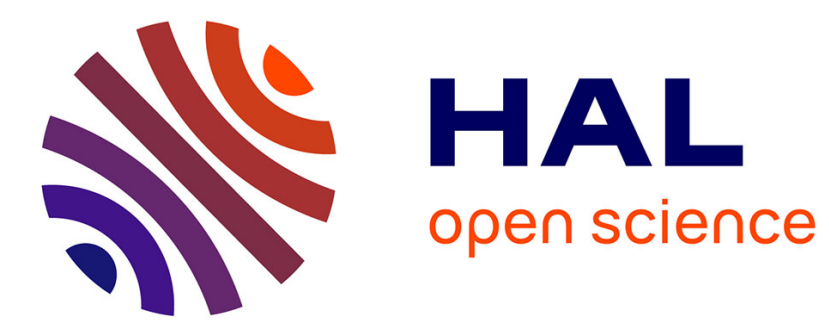

\title{
Invariance-based analysis of cancer chemotherapy
}

Rachid Riah, Mirko Fiacchini, Mazen Alamir

\section{To cite this version:}

Rachid Riah, Mirko Fiacchini, Mazen Alamir. Invariance-based analysis of cancer chemotherapy. MSC 2015 - IEEE Multi-Conference on Systems and Control, Sep 2015, Sydney, Australia. hal-01208074

\section{HAL Id: hal-01208074 \\ https://hal.science/hal-01208074}

Submitted on 2 Oct 2015

HAL is a multi-disciplinary open access archive for the deposit and dissemination of scientific research documents, whether they are published or not. The documents may come from teaching and research institutions in France or abroad, or from public or private research centers.
L'archive ouverte pluridisciplinaire HAL, est destinée au dépôt et à la diffusion de documents scientifiques de niveau recherche, publiés ou non, émanant des établissements d'enseignement et de recherche français ou étrangers, des laboratoires publics ou privés. 


\title{
Invariance-based analysis of cancer chemotherapy ${ }^{\star}$
}

\author{
Rachid Riah $^{1}$, Mirko Fiacchini ${ }^{1}$ and Mazen Alamir ${ }^{1}$
}

\begin{abstract}
In this paper, chemotherapy of cancer is studied. The model chosen to simulate the behaviour of the cancer is based on the normal and the tumor cells evolution. Aggressive chemotherapy leading to tumor contraction must respect the constraint on the patient health, which is the lower admissible bound of the normal cells. The properties of positively invariant set are employed. The domains of attraction, representing all the initial states of patients which could be brought back to the healthy state by respecting the constraint on the patient health, are determined using set theory tools. The domains of attraction corresponding to different chemotherapy profiles can be characterized with this method.
\end{abstract}

Kaywords: Cancer chemotherapy, Invariance, Set theory.

\section{INTRODUCTION}

Cancer is a dangerous disease that kills every year thousands of people in the world. Thus, several scientific researchers and doctors have been attracted to work on this topic, in order to help the patients who suffer from this disease. In the literature, several mathematical models to stimulate the evolution of the cancer cells have appeared in the last decades, some of them are based on the evolution of the different cells, see [1], [7], [12], [17]. Such models may incorporate the effect of external drugs on the evolution of the cancer, as chemotherapy and immunotherapy drug or their combination. In the recent years, mathematical models of cancer have been widely used also for control-based tumoral therapy design, applying optimal control [6], [14], or feedback control, see [2], [5].

Our work in this paper is based on the model inspired by the work of [1], [17]. It represents the evolution of normal and tumor cells, taking into account the effect of chemotherapy drug. In [1], this model is used to represent the evolution of acute myeloblastic leukemia (AML) and in [17] it has been used to simulate the cancer evolution. The normal cells represent a part of the innate immune system of the human body and can be interpreted as the aggregation of NK cells, CD8+ cells and a circulating lymphocytes (or white blood cells) as defined in [5].

In the literature, there are two important categories of analysis, reachability analysis, which computes the set of reachable states when the initial state, input, and parameters are uncertain [16], and the viability kernel is the set of initial states for which there exists an input such that the system respects the state constraint for all time. Typically, the viability kernel is much harder to compute [20], and in this paper, our approach is substantially based on techniques and methods from set theory and invariance for control. The importance of invariant sets in control is due to stability and robustness implicit properties of these regions of the state space. Many results regarding invariance and related topics have been provided in literature: see, for instance, the notable pioneering contribution [3], the works [11], [13], concerning

${ }^{\star}$ This work was supported by the French national project CATSINSERM.

1 GIPSA-lab, Grenoble Campus, 11 rue des Mathématiques, BP 46, 38402 Saint Martin d'Hères Cedex, France. \{rachid.riah, mirko.fiacchini, mazen.alamir\}@gipsa-lab.fr the maximal invariant set, and [18] regarding the minimal one. The problem of obtaining invariant sets for discretetime nonlinear systems is dealt with using ellipsoids in [15] and polytopes in [9]. A recent monograph on invariance and set-theory in control is [4].

The main objective of this paper is to determine the maximal invariant polyhedral set that represents all states of patients that could be forced to converge to the desired healthy states, applying a chemotherapy drug administration strategy. The desired set is defined to be the set for which the number of tumor cells is close to zero and the normal cells higher then the minimal admitted level. A minimal level is imposed as a health condition. This maximal set represents the domain of attraction of the cancer system.

As is proved in [17] that the strategy of aggressive chemotherapy is the best technique to eradicate all the cancer cells, in this preliminary work, the control inputs are considered to take maximum chemotherapy drugs or no chemotherapy drugs.

A comparative study between several drug strategies is also provided. The characterization of the all domains of attraction for each drug control profile is given, and the suitable strategy for each patients can be inferred by analyzing the different domains of attraction.

The paper is organized as follows. In Section II, the mathematical model of cancer is introduced and the problem statement is given. In Section III, the properties and numerical methods related to the positively invariant sets are given. The analysis of an appropriate drug administration strategy, and the comparison among different therapeutic profiles are given in Section IV. Section V finishes the paper with some conclusions and future works.

Notation: Given $n \in \mathbb{N}$, define $\mathbb{N}_{n}=\{x \in \mathbb{N}: 1 \leq x \leq n\}$. Given a matrix $A \in \mathbb{R}^{n \times m}, A_{i}$ with $i \in \mathbb{N}_{n}$ denotes its $i$-th row, $A_{i, j}$ with $i \in \mathbb{N}_{n}$ and $j \in \mathbb{N}_{m}$ denotes the value that corresponds to the position in the matrix $A$ defined by the $i$-th row and the $j$-th column.

\section{MATHEMATICAL CANCER MODEL}

Our main objective is to provide an analytical tool to the biologists for inferring which chemotherapy profile can lead to substantial reduction of the tumor while maintaining a patient health indicator above a threshold. The suitable therapeutic profile should be function of the initial state of the tumor. Thus, the control goal is to steer the tumor at an admissible size at the end of the therapy, by holding the normal cells above the admitted level which is representative of an healthy condition of the patient.

We consider the mathematical model used in [1], [17] that models the interaction between tumoral and normal cells and the effect of chemotherapy drug. This low dimensional model has been used by many researches in the past to reproduce and simulate the cancer brain dynamic. The two dynamics represented in this model are the evolution of the tumor cell populations and the normal cell populations. The interactions 
between them and the effect of chemotherapy drugs are taken account in this model. The model is based on the following assumptions, taken from [17]:

1) The growth dynamics of both tumor and normal cell populations are the same anywhere in the body (i.e. all cells populations are homogeneous).

2) The tumor and normal cells populations follow a process of Gompertizian growth.

3) The chemotherapy drug kills both tumor and normal cells and affects the normal more than the tumor cells.

4) The tumor cells exhibit a negative and inhibiting effect on the growth and development of the normal cells.

5) The drug spreads instantaneously within the body (i.e. the drug amount within the body is proportional to the drug infusion rate).

The resulting model, based on the above assumptions, is defined as follows:

$$
\left\{\begin{array}{l}
\dot{L}=\alpha L \ln \frac{\theta_{L}}{L}-\Pi_{1}(c) L, \quad L(0)=L_{0}, \\
\dot{N}=\beta N \ln \frac{\theta_{N}}{N}-\Xi(L) N-\Pi_{2}(c) N, \quad N(0)=N_{0} .
\end{array}\right.
$$

This model involves 2 states and 1 control input. All variables and parameters are given hereafter:

- $L$ and $N$ are the tumor and the normal cell population respectively,

- $\alpha$ is the tumor growth rate, $\beta$ is the normal growth rate,

- $\theta_{L}$ is the greatest size of the tumor cell population, $\theta_{N}$ is the normal size of the normal cell population,

- $-\Xi(L) N$ represents the negative effect of the tumor on the normal cells,

- $c$ stands for the concentration of the chemotherapy drug,

- $\Pi_{1}(c)$ and $\Pi_{2}(c)$ are the loss functions for both population due to chemotherapy effect.

As in the work [17], we adopt in the sequel the following approximations:

1) the function $\Xi(L)$ is taken to be linear as in [1], [17] (i.e. $\Xi(L)=\gamma L$, where $\gamma$ is a given parameter);

2) we consider that the loss function $\Pi_{1}(c)$ as a control input taking into account the saturation phenomena (i.e, $\Pi_{1}(c)=u$ );

3) we also assume that $\Pi_{2}(c)=l \Pi_{1}(c), l$ is a given parameter (i.e. $\Pi_{2}(c)=l u$ ).

Thus, the resulting model becomes:

$$
\left\{\begin{array}{l}
\dot{L}=\alpha L \ln \frac{\theta_{L}}{L}-u L, \quad L(0)=L_{0}, \\
\dot{N}=\beta N \ln \frac{\theta_{N}}{N}-\gamma L N-\operatorname{lu} N, \quad N(0)=N_{0} .
\end{array}\right.
$$

Physically the normal cells growth faster then the tumor ones. In view of this, we assume that the rate growth of normal cells $\beta$ is bigger then the rate growth of tumor cells $\alpha$, see [1], [17], that is $\beta>\alpha$. Notice that the nonlinear model (2) involves $n_{p}=6$ parameters. The consistent values for these parameters are inferred from the scientific literature and summarized in Table 1, see [1], [17].

\section{A. Equilibrium points and observations}

Notice that the first equation in Eq.(2) has one zero at the tumor free equilibrium at $L_{E}=0$ and several possible non zero tumor equilibria. The tumor free equilibrium for the system is given by $\left(L_{E}, N_{E}\right)=\left(0, \theta_{N}\right)$. Let $E_{0}$ represents the point $\left(0, \theta_{N}\right)$. To determine the equilibria in the case where $L_{E} \neq 0$, we have to solve the Eq.(2) and find the non zero tumor equilibria. This point is $\left(L_{E}, N_{E}\right)=$ $\left(\theta_{L}, \theta_{N} e^{-\frac{\gamma \theta_{L}}{\beta}}\right)$ in absence of chemotherapy drug. According to the Gompertzian dynamics of the tumor cells, the tumor free equilibrium is unstable, while the high tumor equilibrium is stable. This means that if the treatment is stopped, the system returns inevitably to the hight tumor state.

According to the model, when no cancer cells are present in the body, the normal cells grow monotonically and tend toward its asymptotic bound. Tumor cells increase also monotonically with time, in the presence of normal cells. It is asserted that the two cell populations follow a process of Gompertzian growth. Thus, when the cancer is large enough to affect the normal cells, these last cells tend to decrease dramatically to an abnormally low level. This is due to the negative effects exercised by the tumor cells on the normal ones. However, the tumor cells continue to increase to his bound limit and the design of chemotherapy is very complex and should therefore incorporate the aim of slowing down considerably the growth of the tumor cells and removing the inhibition that they exercise over the normal cells [1], [17].

B. Discrete-time model for analysis

First, introducing the change of variables $x_{1}=\ln \frac{\theta_{L}}{L}$ and $x_{2}=\ln \frac{\theta_{N}}{N}$, the equivalent system is

$$
\left\{\begin{array}{l}
\dot{x_{1}}=-\alpha x_{1}+u, \quad x_{1}(0)=x_{10}, \\
\dot{x_{2}}=-\beta x_{2}+\gamma \theta_{L} e^{-x_{1}}+l u, \quad x_{2}(0)=x_{20},
\end{array}\right.
$$

where $x_{10}$ and $x_{20}$ are the initial conditions. The constraints $0 \leq u \leq u_{\max }$ and $x_{2} \leq x_{2 \max }$ are considered, where $u_{\max }$ is the maximal control input and $x_{2 \max }$ is the maximal value of $x_{2}$. Considering that $N_{\min }$ is the minimal admitted level of the normal cells, then $x_{2 \max }=\ln \frac{\theta_{N}}{N_{\min }}$. This change of variables reduces the degree of nonlinearity of the system (2) and permits to better exploit this model.

Finally, the discrete-time system modelling the cancer evolution is obtained by sampling the continuous system (3), with Euler discretization method and sampling time of $T_{s}=$ $0.5 d a y$, opted using Matlab simulations. It results in

$$
\left\{\begin{array}{l}
x_{1}^{+}=\left(1-T_{s} \alpha\right) x_{1}+T_{s} u=f_{1}(x, u), \\
x_{2}^{+}=\left(1-T_{s} \beta\right) x_{2}+T_{s} \gamma \theta_{L} e^{-x_{1}}+T_{s} l u,=f_{2}(x, u), \\
x_{1}(0)=x_{10}, \quad x_{2}(0)=x_{20}
\end{array}\right.
$$

which is more suitable to apply the iterative methods for invariant set computation.

As shown in the literature, the strategy of intensive chemotherapy is the best one to eradicate the cancer cells, [17]. However, this strategy is not efficient for all patients, and in some cases it can worsen the patient's situation by not respecting the health condition. In this paper, our main goal is to determine the domains of attraction of the cancer system related to different chemotherapy profiles. Given a therapeutic strategy, the domain represents all the states of the patients for which the therapy leads to a substantial regression of the tumor size by preventing the health measure to reach values dangerous for the patient. Based on those domains of attraction, the analysis of the more appropriate drug

\begin{tabular}{cccc}
\hline param & value & param & value \\
\hline$\alpha$ & $3.96 \times 10^{-3}$ day $^{-1}$ & $\beta$ & $3.33 \times 10^{-2}$ day $^{-1}$ \\
$\theta_{N}$ & $1.4 \times 10^{12}$ cells $^{-1}$ & $\theta_{L}$ & $3 \times 10^{12}$ cells $^{-6}$ \\
$\gamma$ & $5 \times 10^{-6}(\text { cells.day })^{-1}$ & $l$ & $10^{-2}$ \\
\hline
\end{tabular}

TABLE I: Parameters of the dynamic model (2). 
administration strategy can be inferred, and the comparison between different therapeutic profiles can be performed.

The methods proposed are substantially based on the properties of positively invariant sets that will be introduced in the sequel.

\section{INVARIANCE AND DOMAIN OF ATTRACTION}

The properties of positively invariant sets have been used in the recent years for characterizing the domain of attraction of dynamical systems and are particularly suitable for constrained and robust control. The main aim of this paper is to compute the set of initial values of the number of normal and tumoral cells for which an admissible therapeutic profile exists. This problem consists in practice in obtaining the maximal contractive invariant convex set as an estimation of the domain of attraction for the system (4). Let us now introduce some useful tools to deal with convex closed sets and difference inclusions systems. Consider the difference inclusions system

$$
x^{+} \in \mathcal{F}(x)
$$

where $x \in X \subseteq \mathbb{R}^{n}$ is the state, $x^{+}$is the successor and $\mathcal{F}(\cdot)$ is a set valued map on $\mathbb{R}^{n}$, that is $\mathcal{F}(x)$ represents a function which relates a set to every point $x \in \mathbb{R}^{n}$, i.e. $\mathcal{F}(x) \subseteq \mathbb{R}^{n}$ for all $x \in \mathbb{R}^{n}$. The set $X$ is intended to be, hereafter, the set of state constraints.

Remark 1: The interest of difference inclusions systems relies in the fact that they can be used to represent controlled and/or uncertain systems and also to approximate nonlinear systems $x^{+}=f(x)$, i.e. such that $f(x) \in \mathcal{F}(x)$ for all $x \in$ $X$. In particular, in this paper we use difference inclusions to bound the dynamics of the nonlinear system (4) for $u=0$ and $u=u_{\max }$, and to obtain invariant sets and domain of attraction for the former, that are invariant and domain also for the latter. Surprisingly, the conservatism often introduced when approximating a nonlinear system with a difference inclusions one can be made arbitrarily small for the case under analysis. Thus the exact domain of attraction can be approximated with the desired precision with the present method.

An important tool to deal with convex closed sets is the support function, it is defined hereafter.

Definition 1: Given a set $\Omega \subseteq \mathbb{R}^{n}$, the support function of $\Omega$ evaluated at $\eta \in \mathbb{R}^{n}$ is $\phi_{\Omega} \overline{(\eta)}=\sup _{x \in \Omega} \eta^{T} x$.

Geometrically, the support function of $\Omega$ at $\eta$ is the signed "distance" of the point of the closure of $\Omega$ further from the origin, along the direction $\eta$. See [19] for some properties of support functions. Using the support function is helpful to transform a set-inclusion condition in terms of linear inequalities, see [19] for instance. From the definition of the support function, we get this property.

Property 1: Given two closed, convex sets $\Omega \subseteq \mathbb{R}^{n}$ and $\Gamma \subseteq$ $\mathbb{R}^{n}$, then $x \in \Omega$ if and only if $\eta^{T} x \leq \phi_{\Omega}(\eta)$ for all $\eta \in \mathbb{R}^{n}$, and $\Gamma \subseteq \Omega$ if and only if $\phi_{\Gamma}(\eta) \leq \phi_{\Omega}(\eta)$, for all $\eta \in \mathbb{R}^{n}$.

Before giving the definition of invariance, let us introduce this assumption.

Assumption 1: Assume that the set valued map $\mathcal{F}$ determining the system dynamics (5) is such that $\mathcal{F}(x)$ is compact and convex for all $x \in \mathbb{R}^{n}$ and, for every $\eta \in \mathbb{R}^{n}$, the function $F(x, \eta): \mathbb{R}^{n} \times \mathbb{R}^{n} \longrightarrow \mathbb{R}$ defined as

$$
F(x, \eta)=\phi_{\mathcal{F}(x)}(\eta)=\sup _{z \in \mathcal{F}(x)} \eta^{T} z
$$

is convex with respect to $x$ on $X$.

Thus, in practice, $F(x, \eta)$ is the support function of set $\mathcal{F}(x)$ evaluated at $\eta \in \mathbb{R}^{n}$ and then

$$
\mathcal{F}(x)=\left\{z \in \mathbb{R}^{n}: \eta^{T} z \leq \mathcal{F}(x, \eta), \forall \eta \in \mathbb{R}^{n}\right\},
$$

and it is convex in $x$. The dynamical systems (5) for which Assumption 1 holds are tightly related to the Convex Difference Inclusions (CDI) systems defined in [8], [10].

Remark 2: Notice that, with respect to the CDI systems, no assumption on the value of $F(0, \eta)$ is done here. This because, while the CDI systems defined in [8], [10] are employed for characterizing invariance and contractivity with respect to the origin, in this paper, the convergence of the CDI system is towards the infinity along an asymptote.

Now, we recall the standard definitions of invariance set for the generic nonlinear system, adapted here for the case of potentially unbounded sets that do not necessarily contain the origin and set valued maps.

Definition 2 ([4]): The closed convex set $\Omega \subseteq \mathbb{R}^{n}$ is an invariant set for the system $x^{+}=f(x)$ if $f(x) \in \Omega$, for all $x \in \Omega$, it is invariant for the difference inclusions system (5) if $\mathcal{F}(x) \subseteq \Omega$, for all $x \in \Omega$.

Every trajectory starting in an invariant $\Omega$ remains in it. For the difference inclusions system (5), the invariance of $\Omega \subseteq$ $X$, can be given, as usual, in terms of the one-step backward operator, defined below.

Definition 3: Let Assumptions 1 hold for the set valued map $\mathcal{F}(\cdot)$ determining the dynamic system (5). The one-step operator is defined as

for all $\Omega \subseteq X$.

$$
Q(\Omega)=\{x \in X: \mathcal{F}(x) \subseteq \Omega\},
$$

Hence, the one-step operator associates to every set $\Omega$ the set of points that are mapped inside $\Omega$ through $\mathcal{F}(\cdot)$. Thus, by definition, a closed convex set $\Omega \subseteq X$ is invariant if and only if $\Omega \subseteq Q(\Omega)$ and the one-step operator can be used to check invariance and to compute increasing sequences of nested invariant sets, as for linear or nonlinear systems. Indeed, Algorithm 1, standard for generating increasing invariant approximations of the domain of attraction, see [4], can be applied also in this context. Thus, $\Omega_{k}$ are invariant and

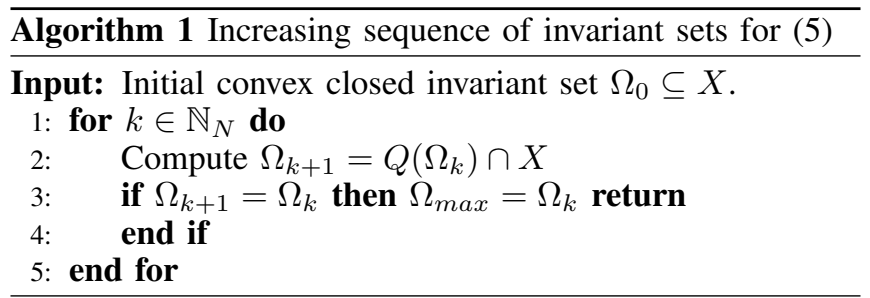

converges to the domain of attraction for the system (5), and hence for all nonlinear systems approximated by (5). Finally, being our objective to have a sequence of $\Omega_{k}$ that are polyhedral, we introduce the following results, proved in [8], which is functional for this purpose.

Proposition 1: Given the set-valued map $\mathcal{F}(x)$ determining the system dynamics (5) and the state constraint set $X$. Given a polytope $\Omega=\left\{x \in \mathbb{R}^{n}: H x \leq h\right\}$, with $H \in \mathbb{R}^{n_{h} \times n}$, the one-step operator is

$$
Q(\Omega)=\left\{x \in X: F\left(x, H_{i}^{T}\right) \leq h_{i}, \forall i \in \mathbb{N}_{n_{h}}\right\} .
$$

Notice that, as proved in [8] for general convex closed sets, for every polytopic $\Omega$, the set $Q(\Omega)$ is closed and convex. Moreover, if $F(\cdot, \eta)$ are piecewise affine functions of $x$, then $Q(\Omega)$ is a polyhedron, that is the intersection of a finite number of halfspaces, for every polytopic (or polyhedral) $\Omega$. Remark 3: The problem of boundedness of the sets $\Omega_{k}$ can be addressed, for computational aims, by adding appropriate fictitious constraints on $X$ such that it results to be bounded. This approach has been used to obtain the domains of attraction illustrated in the following. 
In fact, Algorithm 1 is efficient and scalable to provide the maximal invariant set for all high-dimensional nonlinear systems for which the related one-step operator given by (9) is not hardly computable. Once the set of constraint states $X$ and the initial invariant set $\Omega_{0}$ are selected, Algorithm 1 is fully automatic and no parameters are needed to be selected. This operator is employed to find the region of stability of the system which represents the evolution of the cancer, for the given profile of the chemotherapy drug.

\section{CANCER CHEMOTHERAPY ANALYSIS}

In order to use the Algorithm 1 to find the maximal invariant set for the system (4), reproducing the evolution of the cancer, the one-step operator defined in Proposition 1 is employed. Once the maximal invariant set found in this domain, it will be translated to the tumor-normal cells domain.

Remark 4: From many results in literature, see for instance [17], one can infer that the most efficient drug profiles are given by sequences of periods of maximal delivery, i.e. $u=u_{\max }$, and periods of no chemotherapy drug perfusion, i.e. $u=0$. Hence, in this paper, the profiles composed by maximal or null drug deliverance are considered. Nevertheless, the method proposed can be applied directly for variable values of $u$, it is sufficient to focus on control invariance sets in spite of invariant ones.

To determine the one-step operator for the two dynamics, with $u=u_{\max }$ and $u=0$, it necessary to determine the bounding functions $F_{u}(x, \eta)$ for all $\eta \in \mathbb{R}^{2}$, convex with respect to $x$ and such that the related set valued maps

$$
\mathcal{F}_{u}(x)=\left\{z \in \mathbb{R}^{n}: \eta^{T} z \leq F_{u}(x, \eta), \forall \eta \in \mathbb{R}^{n}\right\},
$$

as (7), satisfies $f(x, u) \in \mathcal{F}_{u}(x)$, with $f(x, u)$ in (4), for every $x \in X$ and every $u \in\left[0, u_{\max }\right]$. From Property 1 , these functions $F_{u}(x, \eta)$ must be convex and such that $\eta^{T} f(x, u) \leq F_{u}(x, \eta)$, for every $\eta \in \mathbb{R}^{n}$. Moreover, since the nonlinearity involves only $f_{2}(x, u)$ then the bounding functions of $\eta^{T} f(x, u)$ are related only to $\eta_{2}$, since the dynamics of $x_{1}$ is linear. A possible choice is

- if $\eta_{2} \geq 0: \quad F_{u}(x, \eta)=\eta^{T} f(x, u)$,

since $F_{u}(x, \eta)$ is already convex in this case;

- if $\eta_{2}<0$ :

$$
\begin{aligned}
F_{u}(x, \eta)= & \eta_{1} f_{1}(x, u)+\eta_{2}\left(\left(1-T_{s} \beta\right) x_{2}\right. \\
& \left.+T_{s} \gamma \theta_{L}\left(a x_{1}+b\right)+T_{s} l u\right),
\end{aligned}
$$

with $a$ and $b$ such that $\left(a x_{1}+b\right) \leq e^{-x_{1}}$, obtained for instance as the tangent to the graph of $e^{-x_{1}}$ at one point and $T_{s}$ is the sampling time;

for all $\eta \in \mathbb{R}^{2}$.

Nevertheless, we are interested in convex piecewise affine bounding functions for computational purposes. For this it is sufficient to replace $e^{-x_{1}}$ with a convex piecewise affine upper bound, which is easily obtainable, for the case of $\eta_{2} \geq 0$. In fact, it is sufficient to choose a set of $p \in \mathbb{N}$ parameters $c_{i} \in \mathbb{R}, d_{i} \in \mathbb{R}$, with $i \in \mathbb{N}_{p}$, such that, $e^{-x_{1}} \leq \max _{i \in \mathbb{N}_{p}}\left\{c_{i} x_{1}+d_{i}\right\}$, for all $x_{1} \in \mathbb{R}$. To obtain those parameters, it is sufficient to define $z_{i}$ with $i \in \mathbb{N}_{p+1}$ such that $z_{i}<z_{i+1}$ and then

$$
c_{i}=\frac{e^{-z_{i+1}}-e^{-z_{i}}}{z_{i+1}-z_{i}}, \quad d_{i}=\frac{z_{i+1} e^{-z_{i}}-z_{i} e^{-z_{i+1}}}{z_{i+1}-z_{i}},
$$

is the affine function such that $e^{-x_{1}} \leq c_{i} x_{1}+d_{i}$ for every $x_{1} \in\left[z_{i}, z_{i+1}\right]$ and $e^{-z_{i}}=c_{i} z_{i}+\bar{d}_{i}$ for all $i \in \mathbb{N}_{p}$, see Figure 1 .
Remark 5: Notice that, in order to increase the precision, the number of parameters $p$ can be taken as big as desired. Hence, arbitrary precision can be attained.

Concerning, the case of $\eta_{2}<0$, no modification is required with respect to (10), being this already piecewise affine, affine in fact. Then, we obtain

$$
\begin{aligned}
& \text { - if } \eta_{2} \geq 0 \text { : } \\
& \begin{aligned}
F_{u}(x, \eta)= & \eta_{1} f_{1}(x, u)+\eta_{2}\left(\left(1-T_{s} \beta\right) x_{2}\right. \\
& \left.+T_{s} \gamma \theta_{L} \max _{i \in \mathbb{N}_{p}}\left\{c_{i} x_{1}+d_{i}\right\}+T_{s} l u\right),
\end{aligned}
\end{aligned}
$$

- if $\eta_{2}<0: F_{u}(x, \eta)$ as in (10).

Figure 1 shows the convex upper bound and the concave lower bound of $e^{-x_{1}}$ implicitly used to determine $F_{u}(x, \eta)$. Notice that $F_{u}(x, \eta)$ is a convex upper bound of $\eta^{T} f(x, u)$, for all $x \in X, \eta \in \mathbb{R}^{n}$ and $u \in\left[0, u_{\max }\right]$.

Remark 6: Since in this application the sequence of polytopes generated have never a facet determined by $H_{i}$ such that $H_{i, 2}<0$ (except the trivial constraints $x_{2} \leq 0$ ), then the lower bound of $e^{-x_{1}}$ is never employed. This would mean that the sequence of polytopes obtained and the domain of attraction are affected only by the mismatches between $e^{-x_{1}}$ and the piecewise function $\max _{i \in \mathbb{N}_{p}}\left\{c_{i} x_{1}+d_{i}\right\}$, mismatch that can be done arbitrarily small, as notice in Remark 5. Hence, the desired precision can be achieved by employing sufficiently close piecewise approximations of $e^{-x_{1}}$.

Therefore, given a polytope $\Omega=\left\{x \in \mathbb{R}^{2}: H x \leq h\right\}$ with $H \in \mathbb{R}^{n_{h}, n}$, and using the upper bound found above and the consideration in Remark 6, then one-step operator defined in the Proposition 1 is

$$
\begin{aligned}
& Q_{u}(\Omega)=\left\{x \in \mathbb{R}^{2}: \forall i \in \mathbb{N}_{n_{h}}, \forall j \in \mathbb{N}_{p},\right. \\
& \quad H_{i, 1} f_{1}(x, u)+H_{i, 2}\left(\left(1-T_{s} \beta\right) x_{2}+T_{s} \gamma \theta_{L} c_{j} x_{1}\right) \\
& \left.\quad \leq h_{i}-H_{i, 2} T_{s} l u-H_{i, 2} T_{s} \gamma \theta_{L} d_{j}\right\},
\end{aligned}
$$

for every $u \in\left[0, u_{\max }\right]$. Once this operator is defined, for every given chemotherapy control profile the related onestep operator can be determined by composing (12). For instance, the one-step operator for the profile given by one sampling period of full drug injection and two period of null chemotherapy is $Q_{\left[u_{\max , 0,0]}\right.}=Q_{u_{\max }}\left(Q_{0}\left(Q_{0}(\Omega)\right)\right)$.

Hence, given a chosen drug delivery profile and an initial invariant polyhedral set for this profile, the Algorithm 1 can be applied to generate an increasing sequence of nested invariant sets converging to the exact domain of attraction for that particular therapy.

Definition 4: The set of healthy states is defined to be all the states of a patients such that the number of a tumor cells is small enough and the number of normal cells is higher then the minimal admitted level. Such set, mapped in the space of $x$, is the set with $x_{1}$ big enough and $x_{2} \leq x_{2 \max }$.

Remark 7: Notice that, the initial invariant polyhedral set $\Omega_{0}$, which is required to start the recursive procedure, is

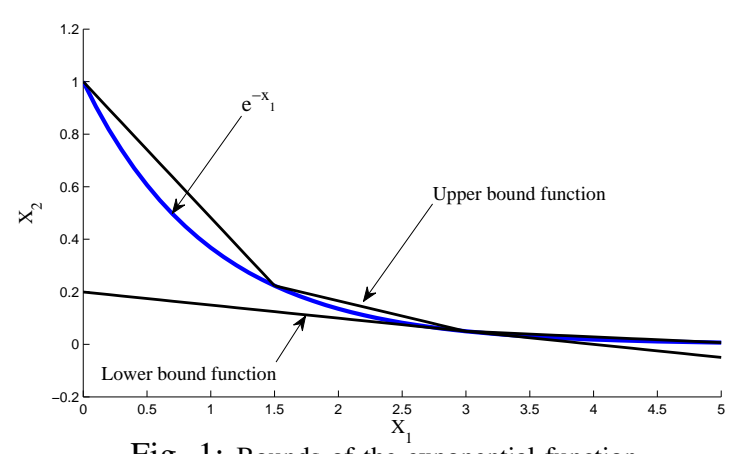

Fig. 1: Bounds of the exponential function. 
taken as a set of healthy states. This initial set has been proved to be invariant for the chemotherapy profiles considered by proving that the related one-step operator provides a set containing $\Omega_{0}$, condition equivalent to invariance.

\section{A. Aggressive chemotherapy}

The first contribution in this paper leads to determine the maximal domain of attraction of the cancer system, which represents all the initial conditions for different patients affected by cancer, that we can bring to a set of healthy states applying an aggressive chemotherapy.

Definition 5: An aggressive chemotherapy is defined to be a therapy that apply heavy doses of chemotherapy drug, i.e. $u(k)=u_{\max }$ for every $k \in \mathbb{N}$.

In this work, we assume that the number of a minimal admitted level of normal cells is $N_{\min }=4.282610^{5} \mathrm{cell} s$, which gives with the change of variable that $x_{2 \max }=$ 15. This level is chosen approximately in order to better show our contribution using simulation. Thus, for clinician experiments the exact value must be employed.

Now, applying the standard recursion starting from the initial invariant set, one gets a sequence of invariant sets that converges to the maximal invariant set for the system (4). Notice that, the same set can be obtained by starting the Algorithm 1 from the set of constraint $X$.

Figure 2 shows the sequences of sets that converge to the exact domain of attraction of the system (4) (except for the piecewise approximation) in bold line and shows that the trajectories of the system with initial states at the vertices of the maximal invariant set converge to the set of healthy states, eradicating the cancer cells without violating the constraint on the patient's health. Theses sets are obtained starting with the initial invariant set. Recall that for every $\Omega_{k}$, the set $\Omega_{k+1}$ contains all states that will be mapped in $\Omega_{k}$ in one step using one heavy dose of drug.

Figure 3 represents the maximal domain of attraction of the cancer system in the space of $N$ and $L$. Notice that, the trajectories of the continuous-time nonlinear system (2) starting from the vertices of the maximal domain of attraction converge toward the set of healthy states.

This domain of attraction represents, with arbitrary precision, the exact set of all the initial patient states that can be cured using aggressive chemotherapy. Thus, given the initial state of a patient, one could infer on the efficacy of the aggressive chemotherapy, on the amount of drug to deliver and on the therapy length.

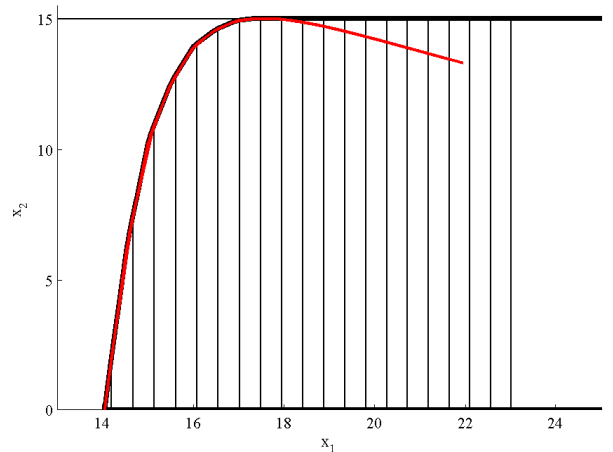

Fig. 2: Maximal invariant set (in black bold line), sequences of polytopes (in black fine lines) and trajectories (in red line) starting from the vertices of the maximal invariant set, for the system (4). The admitted level of $x_{2}$ is equivalent to the admitted level of the normal cells, which is $4.2826 \times 10^{5}$ cells. The chemotherapy drugs $u(k), \forall k \in N$ are taken equal to $u_{\max }$.

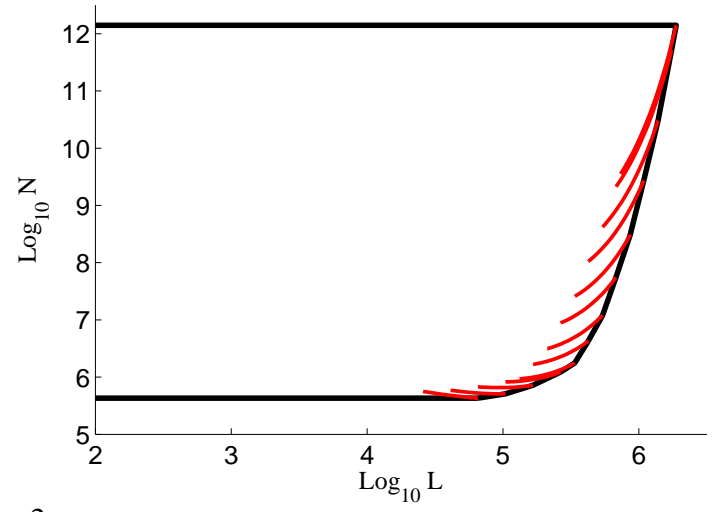

Fig. 3: Maximal domain of attraction of cancer system 2 (in bold black line) and trajectories (in red line) starting from the vertices of the maximal domain of attraction. The admitted level of the normal cell population is $4.2826 \times 10^{5}$ cells. The chemotherapy drugs $u(k), \forall k \in N$ are taken equal to $u_{\max }$.

\section{B. Comparison between therapeutic profiles}

In order to analyse and compare the domain of attraction for an appropriate drug administration strategy, that might be defined a priori by a doctor, three profiles of chemotherapy drugs are taken into account, which are

- Control profile 1: Heavy doses of drugs are applied all the time.

- Control profile 2: Heavy doses of drugs are applied for 3 days then no doses are applied for 3 days.

- Control profile 3: Heavy doses of drugs are applied for 1 days then no doses are applied for 1 days.

Figure 4 shows the three domains of attraction of tumor model given by (2). Thus, depending on the control profile adopted, a specific maximal invariant set is determined. By analyzing the different domains, one can notice that there are initial conditions that cannot be cured by the control profile 3 , whereas they can be healed by applying the control profile 2 . Notice that both profiles 2 and 3 have the same drug delivery rate, i.e. $0.5 u_{\max } / d a y$. Therefore, it is beneficial to attack the cancer cells for long period then release, than for small period.

In Figure 5, a test of invariance for the control profile 3 is presented. As it can be seen in this figure, the boundary states of this set are all states evolve inside the set by applying heavy doses in the first two steps, and then, when no drug is applied, they are mapped to the boundary of the set. This is the behavior that one should expect from the maximal invariant set, that is by the domain of attraction.

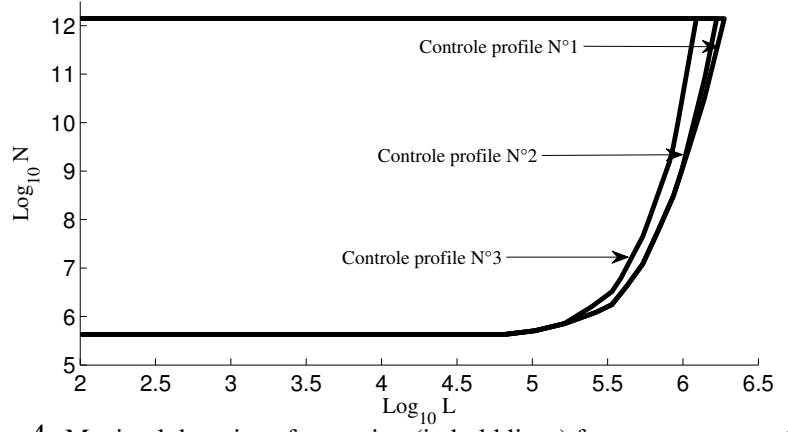

Fig. 4: Maximal domains of attraction (in bold lines) for cancer system, for control profiles 1,2 and 3 . The admitted level of the normal cell population is $4.2826 \times 10^{5}$ cells. 


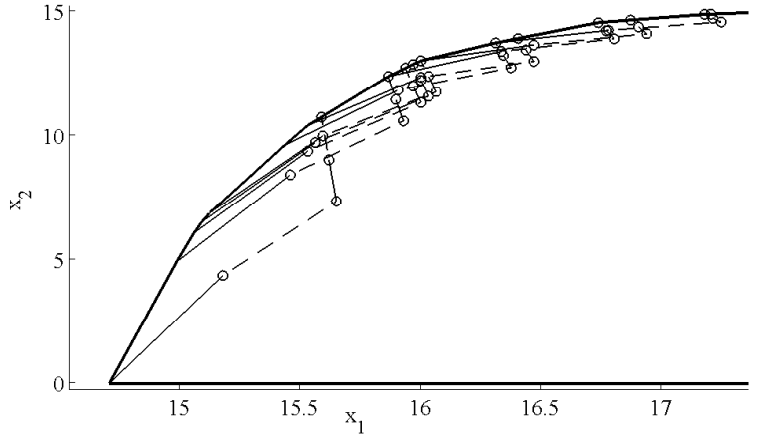

Fig. 5: Maximal domain of attraction for cancer system (in bold line), applying control profile 3 and trajectories (in fine and dashed lines). The admitted level of the normal cell population is $4.2826 \times 10^{5}$ cells.

Now, let us consider and compare the two following profiles

- Control profile 4: No doses of drugs are applied for 2 days then heavy doses are applied for 2 days.

- Control profile 5: Heavy doses of drugs are applied for 2 days then no doses are applied for 2 days.

Figure 6 proves that the set of initial states that can be healed by the control profile 4 is far smaller than the one related to those cured by profile 5 . Noticing that profile 4 is analogous to apply profile 5 after two days of no drug perfusion, one can deduce the reasonable conclusion that delays on the application of a given therapy could undermine substantially its efficiency. This conclusion is in line with the recognized importance of screening and the crucial benefit of early detection of the tumor. From Figures 4, 6, we can see that the aggressive chemotherapy has the greatest domain of attraction among the therapies analyzed here.

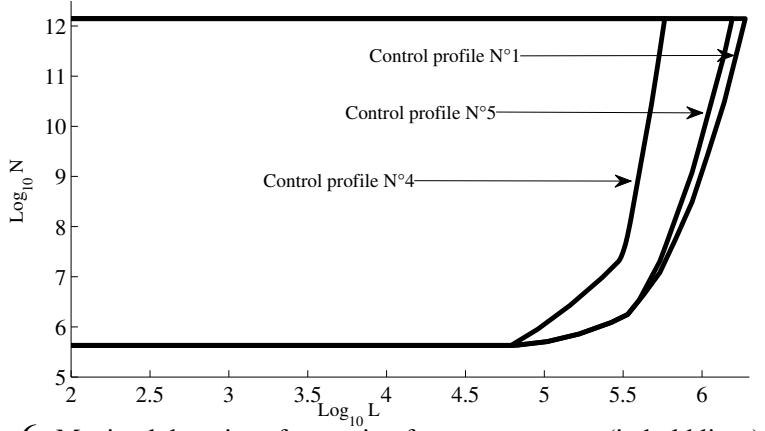

Fig. 6: Maximal domains of attraction for cancer system (in bold lines), for control profiles 1, 4 and 5 . The admitted level of the normal cell population is $4.2826 \times 10^{5}$ cells.

In Figure 7, all the domains for each control profile are showed together. Using this mapping, the appropriate drug strategy can be chosen according to the state of the patient, to the amount of drug to deliver or other constraints.

\section{CONCLUSION}

In this paper, the techniques for computing the domain of attraction for nonlinear systems are applied to determine which are the states of tumor patients that can be successfully cured. The efficiency of different therapeutic profiles can also be inferred by comparing the different domains of attraction. Many directions of future work are open by this preliminary work: control input with all possible value, not only maximum control input or no control input could be considered, the inherent presence of modelling uncertainties, which could also integrate the sampling errors, should be

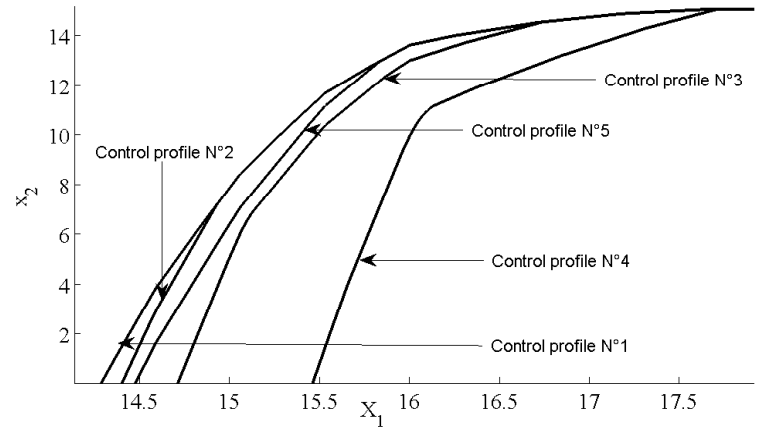

Fig. 7: Maximal invariant sets (in bold line) for the system (4) for control profiles $1,2,3,4$ and 5. The admitted level of $x_{2}$ is equivalent to the admitted level of the normal cells, which is $4.2826 \times 10^{5}$ cells.

taken into account; more complex therapy profiles could be considered; alternative modelling frameworks could be employed, for instance.

\section{REFERENCES}

[1] E. Afenya. Acute leukemia and chemotherapy: a modeling viewpoint. Mathematical biosciences, 138(2):79-100, 1996.

[2] M. Alamir. On probabilistic certification of combined cancer therapies using strongly uncertain models. arXiv preprint arXiv:1502.06218, 2015

[3] D. P. Bertsekas. Infinite-time reachability of state-space regions by using feedback control. IEEE Transactions on Automatic Control, 17:604-613, 1972.

[4] F. Blanchini and S. Miani. Set-Theoretic Methods in Control. Birkhäuser, 2008.

[5] S. Chareyron and M. Alamir. Mixed immunotherapy and chemotherapy of tumors: Feedback design and model updating schemes. Journal of theoretical biology, 258(3):444-454, 2009.

[6] L. G. de Pillis, W. Gu, K. R. Fister, T. Head, K. Maples, A. Murugan, T. Neal, and K. Yoshida. Chemotherapy for tumors: An analysis of the dynamics and a study of quadratic and linear optimal controls. Mathematical Biosciences, 209(1):292-315, 2007.

[7] L. G. de Pillis, W. Gu, and A. E. Radunskaya. Mixed immunotherapy and chemotherapy of tumors: modeling, applications and biological interpretations. Journal of theoretical biology, 238(4):841-862, 2006.

[8] M. Fiacchini. Convex difference inclusions for systems analysis and design. PhD thesis, Universidad de Sevilla, Spain, January 2010.

[9] M. Fiacchini, T. Alamo, and E. F. Camacho. On the computation of convex robust control invariant sets for nonlinear systems. Automatica, 46(8):1334-1338, 2010.

[10] M. Fiacchini, T. Alamo, and E. F. Camacho. Invariant sets computation for convex difference inclusions systems. Systems \& Control Letters, 61(8):819-826, 2012.

[11] E. G. Gilbert and K. Tan. Linear systems with state and control constraints: The theory and application of maximal output admissible sets. IEEE Transactions on Automatic Control, 36:1008-1020, 1991.

[12] P. Hahnfeldt, D. Panigrahy, J. Folkman, and L. Hlatky. Tumor development under angiogenic signaling a dynamical theory of tumor growth, treatment response, and postvascular dormancy. Cancer research, 59(19):4770-4775, 1999.

[13] I. Kolmanovsky and E. G. Gilbert. Theory and computation of disturbance invariant sets for discrete-time linear systems. Mathematical Problems in Engineering, 4:317-367, 1998.

[14] U. Ledzewicz, H. Schättler, and A. d'Onofrio. Optimal control for combination therapy in cancer. In $C D C$, pages 1537-1542, 2008

[15] L. Magni, G. De Nicolao, L. Magnani, and R. Scattolini. A stabilizing model-based predictive control algorithm for nonlinear systems. Automatica, 37:1351-1362, 2001.

[16] A. Matthias, S. Olaf, and B. Martin. Reachability analysis of nonlinear systems with uncertain parameters using conservative linearization. In Proceedings of the 47th IEEE Conference on Decision and Control, pages 4042-4048, 2008.

[17] A. S. Matveev and A. V. Savkin. Application of optimal control theory to analysis of cancer chemotherapy regimens. Systems \& control letters, 46(5):311-321, 2002.

[18] S. V. Raković, E. C. Kerrigan, K. I. Kouramas, and D. Q. Mayne. Invariant approximations of the minimal robust positively invariant set. IEEE Transactions on Automatic Control, 50:406-410, 2005.

[19] R. T. Rockafellar. Convex Analysis. Princeton University Press, USA, 1970

[20] K. Shahab, M. John, O. Meeko, M. Ian M, and D. Guy A. Computing the viability kernel using maximal reachable sets. In Proceedings of the 15th ACM international conference on Hybrid Systems: Computation and Control, pages 55-64. ACM, 2012. 